\title{
Block Renormalization Group for Euclidean Fermions
}

\author{
Tadeusz Balaban, ${ }^{1}$ Michael O'Carroll ${ }^{2}$ and Ricardo Schor ${ }^{2}$ \\ ${ }^{1}$ Mathematics Department, Boston University, Boston, MA, USA \\ ${ }^{2}$ Departmento de Física do ICEx, Universidade Federal de Minas Gerais, Belo Horizonte, \\ Minas Gerais, Brasil
}

\begin{abstract}
Block renormalization group transformations (RGT) for lattice and continuum Euclidean Fermions in d dimensions are developed using Fermionic integrals with exponential and " $\delta$-function" weight functions. For the free field the sequence of actions $D_{k}$ generated by the RGT from D, the Dirac operator, are shown to have exponential decay; uniform in $k$, after rescaling to the unit lattice. It is shown that the two-point function $D^{-1}$ admits a simple telescopic sum decomposition into fluctuation two-point functions which for the exponential weight RGT have exponential decay. Contrary to RG intuition the sequence of rescaled actions corresponding to the " $\delta$-function" RGT do not have uniform exponential decay and we give examples of initial actions in one dimension where this phenomena occurs for the exponenential weight RGT also.
\end{abstract}

\section{Introduction}

Recently rigorous renormalization group methods have been successfully applied to analyze many lattice and continuum models in statistical mechanics and in the Euclidean version of quantum field theory [1-25]. A common feature of the methods applied to the analysis of field theory models in the Euclidean framework is a decomposition of the momentum space achieved by the decomposition of a free continuum propagator or in the case of lattice regularizations by block field transformations. Block renormalization group methods have been used in abelian gauge, abelian gauge-Boson and non-abelian gauge lattice models in two, three and four dimensions to show ultraviolet stability [1-7]. In this paper with a view to future applications to lattice regularized Fermionic and gauge-Fermionic models (see [26-27] for the lattice formulation) we develop the block renormalization group for lattice Fermions. Our treatment parallels the one of [1-3] for scalar and vector Bosons where the RGT is an integral transformation with a Gaussian weight function; for the $\delta$-function weight for the lattice scalar field see [13-14]. The results for the Gaussian case are also similar except for minor changes due to differences in the canonical scaling of free Bosons and Fermions. However there is an unexpected difference in the $\delta$-function formulation. 
As in $[28,29]$ we define the $\operatorname{RGT} T_{a, L}^{\varepsilon}$ acting on a function $\rho(\bar{\psi}, \psi)$ by

$$
\rho^{\prime}(\bar{\chi}, \chi)=\left(T_{a, L}^{\varepsilon} \rho\right)(\bar{\chi}, \chi)=N \int d \bar{\psi} d \psi e^{a(L \varepsilon)^{-1}(\bar{\chi} \cdots Q \bar{\psi}, \ldots-Q \psi)} \rho(\bar{\psi}, \psi),
$$

where $\bar{\chi}, \chi, \bar{\psi}, \psi$ are independent Grassmann algebra generators and carry spinor and lattice indices which have been suppressed. The integral is the Fermionic one of [27]. $\bar{\psi}, \psi(\bar{\chi}, \chi)$ are $\varepsilon(L \varepsilon)$ lattice fields, $Q$ is the arithmetic averaging operator over a block of side size $L \varepsilon$ and $a$ is a positive real parameter. Inner products are always taken as Riemann sum approximations to the continuum. $N$ is a normalization constant, depending on the number of lattice points and spinor components, such that

$$
\int \rho^{\prime}(\bar{\chi}, \chi) d \bar{\chi} d \chi=\int \rho(\bar{\psi}, \psi) d \bar{\psi} d \psi
$$

All integrals are taken in finite volume, the thermodynamic limit is trivial for the sequence of actions treated in this paper and will be implied without further notice.

Applying successive RGT, denoted by $T_{a . L}^{L \varepsilon}, \ldots$ we find the composition law

$$
T_{a, L}^{L^{k-1} \varepsilon} \cdots T_{a, L}^{L \varepsilon} T_{a, L}^{\varepsilon}=T_{a_{k}, L}^{\varepsilon} k,
$$

where the transformation $T_{a_{k}, L}^{\varepsilon} k$ is defined as in (1.1) but with $a_{k}=\left(1-L^{-1} \cdot 1-L^{-k}\right)$ a replacing $a, L^{k}$ replacing $L$, and $Q_{k}$ replacing $Q \cdot Q_{k}$ is the averaging operator over blocks of side size $L^{k} \varepsilon$ and we will use the same symbol, irrespective of the domain lattice, for the average over the $L^{k d}$ points of the block.

In particular if the original density is $e^{(\psi, D \psi)}$, where $D$ is the $\varepsilon$-lattice version of [28] of the Dirac operator

$$
D=\sum_{\mu=1}^{d} \gamma_{\mu}\left(\frac{\partial_{\mu}^{\varepsilon}-\partial_{\mu}^{\varepsilon *}}{2}\right)-\frac{1}{2} \varepsilon \Delta^{\varepsilon}, \Delta^{\varepsilon}=\sum_{\mu=1}^{d} \frac{1}{\varepsilon}\left(\partial_{\mu}^{\varepsilon}+\partial_{\mu}^{\varepsilon *}\right)
$$

then applying the RGT's we get a sequence of $L^{k} \varepsilon$ lattice actions $D_{k}, k=1,2, \ldots$ given by

$$
D_{k}=a_{k}\left(L^{k} \varepsilon\right)^{-1}\left(I+a_{k}\left(L^{k} \varepsilon\right)^{-1} Q_{k} D^{-1} Q_{k}^{*}\right)^{-1} .
$$

In Eq. (1.4) $\partial_{\mu}^{\varepsilon}$ is the $\varepsilon$-lattice forward lattice derivative, $\partial_{\mu}^{\varepsilon *}$ its adjoint, and $\gamma_{\mu}$ are Euclidean self-adjoint Dirac matrices obeying $\gamma_{\mu} \gamma_{\nu}+\gamma_{\nu} \gamma_{\mu}=2 \delta_{\mu \nu}$. The additional non- $\gamma$ terms in Eq. (1.4) are added to suppress the period doubling present in the first term and vanish in the $\varepsilon \rightarrow 0$ limit since $\Delta^{\varepsilon} \stackrel{\varepsilon \rightarrow 0}{\longrightarrow} \sum_{\mu=1}^{d} \partial_{\mu}^{2}$. It will be shown that the $D_{k}$ 's have exponentially decaying kernels.

An important consequence of the composition law (1.3) is that it gives a product decomposition of the free field generating function

$$
e^{-\left(\bar{J}, D^{-1} J\right)}=\int D \bar{\psi} D \psi e^{(\bar{\psi}, D \psi)} e^{(\bar{J}, \psi)+(\bar{\psi}, J)} /(\bar{J}=J=0),
$$

and a sum decomposition of $D^{-1}$, where $\bar{J}$ and $J$ are Grassmann variables. It turns out that the overall structure of the decomposition of the two-point function $D^{-1}$ is trivial; the decomposition is the telescopic sum (letting $D_{0}=D$ and $Q_{0}=I$ )

$$
D^{-1}=\sum_{j=0}^{k-1}\left[D^{-1} Q_{j}^{*} D_{j} Q_{j} D^{-1}-D^{-1} Q_{j+1}^{*} D_{j+1} Q_{j+1} D^{-1}\right]+D^{-1} Q_{k}^{*} D_{k} Q_{k} D^{-1}
$$


which can be written

$$
D^{-1}=\sum_{j=0}^{k-1} S_{j} \Gamma_{j} \bar{S}_{j}^{*}+S_{k} D_{k}^{-1} \bar{S}_{k}^{*}
$$

where $S_{i}=D^{-1} Q_{i}^{*} D_{i}, \bar{S}_{i}^{*}=D_{i} Q_{i} D^{-1}$ are $L^{j} \varepsilon(\varepsilon)$ to $\varepsilon\left(L^{j} \varepsilon\right)$ operators and $\Gamma_{j}=$ $D_{j}^{-1}-D_{j}^{-1} Q^{*} D_{j+1} Q D_{j}^{-1}=\left(D_{j}-a\left(L^{j+1} \varepsilon\right)^{-1} Q^{*} Q\right)^{-1} a L^{j} \varepsilon$ lattice operator. The algebraic structure of (1.5-6) is the same as in the scalar case and is not apparent in [1] but follows from algebraic relations to be derived here; it is apparent in the $a \rightarrow \infty$ limit in [13]. $\Gamma_{j}$ has the interpretation of a fluctuation field two-point function as will be seen below. We will show that the kernels of $S_{j}, \bar{S}_{J}, \Gamma_{j}$ have exponential decay so that roughly speaking

$$
S_{j} \Gamma_{j} \bar{S}_{j}^{*}\left(x, x^{\prime}\right) \sim \frac{1}{\left(L^{j} \varepsilon\right)^{d-1}} e^{-\left(1 / L^{j} \varepsilon\right)\left|x-x^{\prime}\right|}, \quad x, x^{\prime} \varepsilon \varepsilon Z^{d}
$$

Thus (1.5) provides a decomposition into momentum scales $\left(L^{j} \varepsilon\right)^{-1}$.

By the standard procedure of exponentiating $\left(J, D^{-1} J\right)$ given by Eq. (1.6), representing each factor by a Fermionic integral and differentiating with respect to $\bar{J}$ and $J$ we arrive at the following formula for the integral of a function $f(\bar{\psi}, \psi)$ :

$$
\begin{gathered}
\int d \bar{\psi} d \psi e^{(\bar{\psi}, D \psi)} f(\bar{\psi}, \psi) /(f=1)=\int D \bar{\psi}_{k} D \psi_{k} e^{\left(\bar{\psi}_{k}, D_{k} \psi_{k}\right)} \prod_{j=k}^{0} \int D \bar{\eta}_{j} D \eta_{j} e^{\left(\bar{\eta}_{j}, \Gamma_{j}^{-1} \eta_{j}\right)} . \\
f\left(\bar{\psi}=\bar{S}_{k} \bar{\psi}_{k}+\sum_{j=0}^{k-1} \bar{S}_{j} \bar{\eta}_{j}, \psi=S_{k} \psi_{k}+\sum_{j=0}^{k-1} S_{j} \eta_{j}\right) /(f=1)
\end{gathered}
$$

In Eq. (1.8) $\bar{\psi}_{k}, \psi_{k}, \bar{\eta}_{j}, \eta_{j}$ are Grassmann variables. We interpret $\psi_{k}$ as a new field and the $\eta_{j}$ 's as fluctuation fields which have exponential decay. Equation (1.8) can be used as the starting point of a RG analysis, successively integrating over the fluctuation fields.

Equation (1.8) can also be obtained from the RG composition formula by making successive changes of variables in the integral in the left-hand side of Eq. (1.3). The change of variables is devised to eliminate cross terms in the quadratic form and give rise to one step operators. The resulting $j$-step operators, $S_{j}, \bar{S}_{j}$, which have the same structure as the $j$-step minimizers in the scalar field case $[13,14]$, can be written in the form here by using algebraic identities to be derived in Sect. II.

In addition to the RGT (1.1) we can also define directly a RGT which has the properties analogous to a $\delta$-function weight in the scalar case and which is the same as the $a \rightarrow \infty$ limit for the exponential weight. The limiting sequence of actions are given by $\left(Q_{k} D^{-1} Q_{k}^{*}\right)^{-1}$.

However, contrary to $\mathrm{RG}$ intuition, the sequence of actions rescaled to the unit lattice do not have uniform in $k$ exponential decay. We give examples of actions in one dimension where this occurs also for the exponential weight RGT.

Furthermore the decomposition Eq. (1.5) can be carried over to the continuum by taking the $\varepsilon \rightarrow 0, k \rightarrow \infty$ limit such that $L^{k} \varepsilon=1$ which gives, denoting the continuum Dirac operator by $D_{c}=\sum_{\mu} \gamma_{\mu} \partial_{\mu}$, 


$$
\begin{aligned}
D_{c}^{-1}= & \sum_{n=1}^{\infty}\left[D_{c}^{-1} C_{-{ }_{n}}^{*} D^{L^{-n}} C_{-n} D_{c}^{-1}-D_{c}^{-1} C_{-(n-1)}^{*} D^{L-(n-1)} C_{-(n-1)} D_{c}^{-1}\right] \\
& +D_{c}^{-1} C_{0}^{*} D^{1} C_{0} D_{c}^{-1} .
\end{aligned}
$$

In (1.9) $C_{m}: L^{2}\left(R^{d}\right) \rightarrow l_{2}\left(L^{m} Z^{d}\right)$ is a continuum averaging operator over blocks of side length $L^{m}$ and $D^{L^{-m}}=a_{\infty} L^{m}\left(I+a_{\infty} L^{m} C_{-m} D_{c}^{-1} C_{-m}^{*}\right)^{-1}$ is an action on the $L^{-m}$ lattice. Continuing the decomposition in (1.9) to blocks of side $L, L^{2}, \ldots$ we obtain

$$
D_{c}^{-1}=\sum_{m=-\infty}^{\infty}\left[D_{c}^{-1} C_{m}^{*} D^{L^{m}} C_{m} D_{c}^{-1}-D_{c}^{-1} C_{m+1}^{*} D^{L^{(m+1)}} C_{m+1} D_{c}^{-1}\right]
$$

which gives a decomposition of $D_{c}^{-1}$ into space scales $\ldots L^{-1}, 1, L, \ldots$ or the corresponding approximate momentum scales $\ldots L^{1}, 1, L^{-1}, \ldots$.

To make contact with the continuum scalar field decomposition of $[31,32]$ we note that Eq. (1.10) remains valid for $\Delta_{c}$ replacing $D_{c}$ and $\Delta^{L^{m}}=\left(C_{m} \Delta_{c}^{-1} C_{m}^{*}\right)^{-1}$ replacing $D^{L^{m}}$, where $\Delta_{c}$ is minus the continuum Laplacian. Thus

$$
\Delta_{c}^{-1}=\sum_{m=-\infty}^{\infty}\left[\Delta_{c}^{-1} C_{m}^{*} \Delta^{L^{m}} C_{m} \Delta_{c}^{-1}-\Delta_{c}^{-1} C_{m+1}^{*} \Delta^{L m^{+1}} C_{m+1} \Delta_{c}^{-1}\right]
$$

which is the continuum and $a \rightarrow \infty$ limit of the scalar field decomposition of [1]. Applying (1.11) to a function $\Delta_{c} g$ we obtain

$$
g=\sum_{m=-\infty}^{\infty} g_{m}, \quad g_{m} \equiv\left[\Delta_{c}^{-1} C_{m}^{*} \Delta^{L^{m}} C_{m}-\Delta_{c}^{-1} C_{m+1}^{*} \Delta^{L^{m+1}} C_{m+1}\right] g
$$

and simple calculations show that (note $C_{m} C_{m}^{*}=I$ )

$\left(g_{m}, \Delta_{c} g_{n}\right)_{L^{2}\left(R^{d}\right)}=0, m \neq n$, orthogonality;

$C^{n+1} g_{n}=0$, i.e. the average over an $L^{n+1}$ block is zero;

$\Delta_{c} g_{n}$ is constant on $L^{n}$ blocks, i.e. is in the range of $C_{n}^{*}$.

In this way (1.11) provides a decomposition of a function into orthogonal functions in the $\left(\cdot, \Delta_{c} \cdot\right)_{L^{2}\left(R^{d}\right)}$ norm. Of course an analogous decomposition will yield an orthogonal set for any Sobolev norm. In particular for $\Delta_{c}$ replaced by $I g_{n}$ is constant on $L^{n}$ blocks.

We describe the organization of this paper. In Sect. II we obtain expressions for various operators resulting from RGT and obtain various identities including (1.5). Also we define the " $\delta$-function" RGT. In Sect. III we show uniform in $k$ exponential decay of the kernels of the suitably rescaled versions of the operators $D_{k}, \Gamma_{j}, S_{j}, \bar{S}_{j}$. Our proof of decay uses explicit momentum space representations and is more difficult than the scalar case which uses positivity properties of the negative Laplacian (see $[1,13]$ ). In Sect. IV we show the non-uniformity of exponential decay in the case of the $\delta$-function RGT and in one dimension give examples of initial actions which also display this phenomena for the exponential RGT. Section V is devoted to concluding remarks.

\section{Renormalization Group Transformations and Properties}

In this section we establish as in [1-3] the composition law for the RGT and obtain various RGT identities. Additional identities obtained here also have their 
analogs in the scalar case treated in [1]. The decomposition of the two-point function is obtained. Various operator inverses will appear here and will be shown to exist in the next section. We use the more complete notation $D^{(k), L^{k} \varepsilon}$ and $\Gamma^{(k), L^{k_{\varepsilon}}}$ for the $L^{k} \varepsilon$ - lattice actions and $L^{k} \varepsilon$ - lattice fluctuation field two-point functions, respectively. Throughout we use the easily derived relation $Q_{k} Q_{k}^{*}=I, \int d \bar{\psi} d \psi e^{(\bar{\psi}, A \psi)}=$ $\operatorname{det} A$, the translation formula $\int f(\bar{\psi}+\bar{X}, \psi+X) d \bar{\psi} d \psi=\int f(\bar{\psi}, \psi) d \bar{\psi} d \psi$, and $\left.\int d \bar{\psi}, d \psi \exp [\bar{\psi}, A \psi)+(\bar{J}, \psi)+(\bar{\psi}, J)\right]=\operatorname{det} A e^{-\left(\bar{J}, A^{-1} J\right)}$. Here and in the sequel newly introduced field variables are Grassmann and taken to anti-commute with all others.

The composition law is given by

Lemma II.1. $T_{a, L}^{L^{k_{\varepsilon}}} T_{a_{k}, L^{k}}^{\varepsilon}=T_{a_{k+1}, L^{k+1}}^{\varepsilon}$, or in iterated form

$$
T_{a, L}^{L^{k-1} \varepsilon} \cdots T_{a . L}^{L \varepsilon} T_{a, L}^{\varepsilon}=T_{a_{k}, L^{k}}^{\varepsilon},
$$

where $\left(1 / a_{k+1}\right)=\left(L^{-1} / a_{k}\right)+1 / a$ with solution $a_{k}=\left(a\left(1-L^{-1}\right) /\left(1-L^{-k}\right)\right)$.

Proof of Lemma II.1. Using $\bar{\psi}, \psi(\bar{\phi}, \phi)$ for the integration variables of $T_{a, L}^{L^{k_{\varepsilon}}}\left(T_{a_{k}, L^{k}}^{\varepsilon}\right)$ and the translation formula $\int d \bar{\psi} d \psi f(\bar{\psi}, \psi)=\int d \bar{\psi} d \psi f\left(\bar{\psi}+Q_{k} \bar{\phi}, \psi+Q_{k} \phi\right)$, do the $\psi$ integral, use $Q Q^{*}=I$, and equate the coefficients of the quadratic form on both sides.

Define $Z^{(k), L^{k} \varepsilon}$ and $D^{(k+1), L^{k+1} \varepsilon}$ inductively by

$$
\left.Z^{(k), L^{k_{\varepsilon}}} e^{\left(\bar{\psi}, D^{(k+1), L k+1_{k}}\right.} \psi\right) \equiv T_{a, L}^{L^{k_{\varepsilon}}}\left(e^{\left(\bar{\phi}, D^{(k), L k \varepsilon} \phi\right)}\right)
$$

and let

$$
G_{k}^{\varepsilon} \equiv\left(D^{(0), \varepsilon}+a_{k}\left(L^{k} \varepsilon\right)^{-1} Q_{k}^{*} Q_{k}\right)^{-1} .
$$

Lemma II.2. $D^{(k), L^{k} \varepsilon}=a_{k}\left(L^{k} \varepsilon\right)^{-1} I-a_{k}^{2}\left(L^{k} \varepsilon\right)^{-2} Q_{k} G_{k}^{\varepsilon} Q_{k}^{*}$.

Proof of Lemma II.2. By the inductive definition of $D^{(k), L^{k} \varepsilon}$ and the composition $T_{a, L}^{L^{k-1}} \cdots T_{a, L}^{L \varepsilon} T_{a, L}^{\varepsilon}=T_{a_{k}, L^{k}}^{\varepsilon}$, we have $Z_{k}^{\varepsilon} e^{\left(\bar{\psi}, D^{(k), L^{k}} \psi(\psi)\right.}=T_{a_{k}, L^{k}}^{\varepsilon}\left(e^{\left(\phi, D^{(0), \phi_{\phi)}}\right.}\right)$. Performing the integral gives the result.

Define a fluctuation two-point function by the quadratic form in the integral in $T_{a, L}^{L^{k_{\varepsilon}}}\left(e^{\left(\bar{\phi}, D^{\left.(h), L^{k_{\varepsilon}} \phi\right)}\right.}\right)$ by

$$
\Gamma^{(k) \cdot L^{k_{\varepsilon}}} \equiv\left(D^{(k), L^{k_{\varepsilon}}}+a\left(L^{k+1} \varepsilon\right)^{-1} Q^{*} Q\right)^{-1} .
$$

Using $\Gamma^{(k), L^{k_{\varepsilon}}}$ we establish a recursive relation for $D^{(k+1), L^{k_{\varepsilon}}}$ from Eq. (2.1) given by

Lemma II.3. $D^{(k+1) \cdot L^{k+1} \varepsilon}=a\left(L^{k+1} \varepsilon\right)^{-1}-a^{2}\left(L^{k+1} \varepsilon\right)^{-2} Q \Gamma^{(k), L^{k}} Q^{*}$.

We use the definitions and composition formula to find the connection between the operators $G_{k}^{\varepsilon}$ and $\Gamma^{(k), L^{k}}$. By calculation we find

$$
\begin{aligned}
& T_{a_{k}, L^{k}}^{\varepsilon}\left(e^{\left(\bar{\phi}, D^{(0), \varepsilon_{\phi}} \phi\right)+(\bar{\phi}, f)+(\bar{f}, \phi)}\right)(\bar{\psi}, \psi, \bar{f}, f) \\
& \equiv Z_{k}^{\varepsilon} e^{\left(\psi, D^{(k), L_{k} k} \psi\right)} e^{a_{k}\left(L^{k} \varepsilon\right)^{-1}\left[\left(\bar{\psi}, Q_{k} G_{k}^{\varepsilon} f\right)+\left(\bar{f}, G_{k}^{\varepsilon} Q_{k}^{*} \psi\right)\right]} e^{-\left(\bar{f}, G_{k}^{\varepsilon} f\right)},
\end{aligned}
$$

which defines $Z_{k}^{\varepsilon}$. Writing the above for $k+1$ and substituting $T_{a, L}^{L^{k} \varepsilon} T_{a_{k}, L^{k}}^{\varepsilon}$ for $T_{a_{k+1}, L^{k+1}}^{\varepsilon}$ gives rise to important $\mathrm{RG}$ identities given in 


\section{Theorem II.1.}

a) $Z_{k+1}^{\varepsilon}=Z_{k}^{\varepsilon} Z^{(k), L^{k} \varepsilon}$, or in iterated form

$$
Z_{k}=Z^{(k-1) \cdot L^{k-1}} \varepsilon \cdots Z^{(1), L \varepsilon} Z^{(0), \varepsilon} .
$$

b) $a_{k+1}\left(L^{k+1} \varepsilon\right)^{-1} Q_{k+1} G_{k+1}^{\varepsilon}=a\left(L^{k+1} \varepsilon\right)^{-1} a_{k}\left(L^{k} \varepsilon\right)^{-1} Q \Gamma^{(k), L^{k}} Q_{k} G_{k}^{\varepsilon}$,

$$
a_{k+1}\left(L^{k+1} \varepsilon\right)^{-1} G_{k+1}^{\varepsilon} Q_{k+1}^{*}=a\left(L^{k+1} \varepsilon\right)^{-1} a_{k}\left(L^{k} \varepsilon\right)^{-1} G_{k}^{\varepsilon} Q_{k}^{*} \Gamma^{(k), L^{k} \varepsilon} Q^{*} .
$$

c) $G_{k+1}^{\varepsilon}=a_{k}^{2}\left(L^{k} \varepsilon\right)^{-2} G_{k}^{\varepsilon} Q_{k}^{*} \Gamma^{(k) \cdot L^{k}} Q_{k} G_{k}^{\varepsilon}+G_{k}^{\varepsilon}$, or in iterated form

$$
G_{k}^{\varepsilon}=\sum_{j=1}^{k-1} a_{j}^{2}\left(L^{j} \varepsilon\right)^{-2} G_{j}^{\varepsilon} Q_{j}^{*} \Gamma^{(j), L^{\jmath} \varepsilon} Q_{j} G_{j}^{\varepsilon}+\Gamma^{(0), \varepsilon} .
$$

We now establish other representations for $G_{k}^{\varepsilon}, D^{(k), L^{k} \varepsilon}, \Gamma^{(k), L^{k} \varepsilon}, G_{j}^{\varepsilon} Q_{j}^{*}$ and $Q_{j} G_{j}^{\varepsilon}$. These make the $a \rightarrow \infty$ limit more transparent and lead to the decomposition formula. We have

\section{Lemma II.4.}
a) $G_{k}^{\varepsilon}=D^{(0), \varepsilon-1}-a_{k}\left(L^{k} \varepsilon\right)^{-1} D^{(0), \varepsilon-1} Q_{k}^{*}\left(I+a_{k}\left(L^{k} \varepsilon\right)^{-1} Q_{k} D^{(0), \varepsilon-1} Q_{k}^{*}\right)^{-1} Q_{k} D^{(0), \varepsilon-1}$$$
=D^{(0), \varepsilon-1} D^{(0), \varepsilon-1} Q_{k}^{*} D^{(k), L^{k}} Q_{k} D^{(0), \varepsilon-1} \text {. }
$$
b) $D^{(k), L^{k} \varepsilon}=a_{k}\left(L^{k} \varepsilon\right)^{-1}\left(I+a_{k}\left(L^{k} \varepsilon\right)^{-1} Q_{k} D^{(0) \cdot \varepsilon-1} Q_{k}^{*}\right)^{-1}$.
c) $D^{(k+1), L^{k+1} \varepsilon}=a\left(L^{k+1} \varepsilon\right)^{-1}\left(I+a\left(L^{k+1} \varepsilon\right)^{-1} Q D^{(k), L^{k} \varepsilon-1} Q^{*}\right)^{-1}$.
d) $\Gamma^{(k), L^{k} \varepsilon}=D^{(k), L^{k}-1}-D^{(k), L^{k_{\varepsilon}-1}} Q^{*} D^{(k+1), L^{k+1} \varepsilon} Q D^{(k), L^{k_{\varepsilon}-1}}$.
e) $a_{k}\left(L^{k} \varepsilon\right)^{-1} G_{k}^{\varepsilon} Q_{k}^{*}=D^{(0), \varepsilon-1} Q_{k}^{*} D^{(k), L^{k} \varepsilon}$.
f) $a_{k}\left(L^{k} \varepsilon\right)^{-1} Q_{k} G_{k}^{\varepsilon}=D^{(k), L^{k} \varepsilon} Q_{k} D^{(0), \varepsilon-1}$.
g) $D^{(0), \varepsilon-1} Q_{k+1}^{*} D^{(k+1), L^{k+1} \varepsilon}=a\left(L^{k+1} \varepsilon\right)^{-1} D^{(0), \varepsilon-1} Q_{k}^{*} D^{(k), L^{k} \varepsilon} \Gamma^{(k), L^{k}} Q^{*}$, $a\left(L^{k+1} \varepsilon\right)^{-1} \Gamma^{(k), L^{k} \varepsilon} Q^{*}=D^{(k), L^{k} \varepsilon^{-1}} Q^{*} D^{(k+1), L^{k+1} \varepsilon}$.
h) $D^{(k+1), L^{k+1} \varepsilon} Q_{k+1} D^{(0), \varepsilon-1}=a\left(L^{k+1} \varepsilon\right)^{-1} Q \Gamma^{(k), L^{k} \varepsilon} D^{(k), L^{k}} Q_{k} D^{(0), \varepsilon-1}$, $a\left(L^{k+1} \varepsilon\right)^{-1} Q \Gamma^{(k), L^{k} \varepsilon}=D^{(k+1), L^{k+1}} Q D^{(k), L^{k} \varepsilon^{-1}}$.

\section{Proof of Lemma. II.4}

a) Letting $a^{\prime}=a_{k}\left(L^{k} \varepsilon\right)^{-1}$ and multiplying $\left(D^{(0), \varepsilon}+a^{\prime} Q_{k}^{*} Q_{k}\right) f=g$ on the left by $Q_{k} D^{(0), \varepsilon-1}$ gives $Q_{k} f=\left(I+a^{\prime} Q_{k} D^{(0), \varepsilon-1} Q_{k}^{*}\right)^{-1} Q_{k} D^{(0), \varepsilon-1} g$. Substituting for $Q_{k} f$ above gives the first equality of the lemma; the $2^{\text {nd }}$ follows from $b$ );

b) Substitute $G_{k}^{\varepsilon}$ from the first line of Lemma II.4 a) in Lemma II.2 and simplify.

c) Using Lemma II.3. for $D^{(k+1), L^{k_{\varepsilon}}}$ and $\Gamma^{(k), L^{k_{\varepsilon}}}=\left(D^{(k), L^{k_{\varepsilon}}}+a\left(L^{k+1} \varepsilon\right)^{-1} Q^{*} Q\right)^{-1}$, the result follows as in the proof of b),

d) From $\Gamma^{(k), L^{k} \varepsilon}=\left(D^{(k), L^{k} \varepsilon}+a\left(L^{L+1} \varepsilon\right)^{-1} Q^{*} Q\right)^{-1}$ and as in the proof of a) the result follows using Lemma II.4c.

e) Use Lemma II.4 a) for $G_{k}^{\varepsilon}$ and simplify.

f) The result follows using Lemma II.4b similar to e).

g) and h) express Theorem II.1b) in terms of Lemmas II.4e) and f).

We now show that in the decomposition identity of Theorem II.1c for $G_{k}^{\varepsilon}$ that the sum is actually telescopic. 
Theorem II.2.

$$
\begin{aligned}
a_{j}^{2}\left(L^{j} e\right)^{-2} G_{j}^{\varepsilon} Q_{j}^{*} \Gamma^{(j), L^{j} \varepsilon} Q_{j} G_{j}^{\varepsilon}= & D^{(0), \varepsilon-1} Q_{j}^{*} D^{(j), L^{j} \varepsilon} Q_{j} D^{(0), \varepsilon-1} \\
& -D^{(0), \varepsilon-1} Q_{j+1}^{*} D^{(j+1), L^{j+1}} Q_{j+1} D^{(0), \varepsilon-1} .
\end{aligned}
$$

Proof of Theorem II.2 follows from Lemma II.4 d)-f).

We immediately obtain from Theorem II.1c, using Lemma II.4a for $G_{k}^{\varepsilon}$, the decomposition formula

$$
\begin{aligned}
D^{(0), \varepsilon-1}= & \sum_{j=0}^{k-1}\left(D^{(0), \varepsilon-1} Q_{j}^{*} D^{(j), L^{j} \varepsilon} Q_{j} D^{(0), \varepsilon-1}-D^{(0), \varepsilon-1} Q_{j+1}^{*} D^{(j+1), L^{j+1}} Q_{j+1} D^{(0), \varepsilon^{-1}}\right) \\
& +D^{(0), \varepsilon^{-1}} Q_{k}^{*} D^{(k), L^{k} \varepsilon} Q_{k} D^{(0), \varepsilon-1} .
\end{aligned}
$$

We note that the specific form of the individual terms can also be obtained by telescoping $G_{k}^{\varepsilon}$ as given by Lemma II.4a.

Formally the $a \rightarrow \infty$ limit of $D^{(k), L^{k} \varepsilon}$ is, from Lemma II. $4 \mathrm{~b},\left(Q_{k} D^{(0), \varepsilon-1} Q_{k}^{*}\right)^{-1}$ and the $a \rightarrow \infty$ limits of the relations in Lemma II.4c)-f) and also Theorem II.2 are clear.

\section{Fundamental Sequences of Operators and Decay Properties}

In this section we show the exponential decay of $G_{k}^{\varepsilon}, D^{(k), L^{k} \varepsilon}, \Gamma^{(k), L^{k_{\varepsilon}}}, D^{(0), \varepsilon-1} Q_{k}^{*} D^{(k), L^{k_{\varepsilon}}}$ and $D^{(k), L^{k} \varepsilon} Q_{k} D^{(0), \varepsilon-1}$. It is convenient to introduce rescaled operators

$$
\begin{aligned}
& G_{k}=\left(D^{\eta}+a_{k} Q_{k}^{*} Q_{k}\right)^{-1}, \\
& D^{(k)}=a_{k} I-a_{k}^{2} Q_{k} G_{k} Q_{k}^{*}, \\
& \Gamma^{(k)}=\left(D^{(k)}+a L^{-1} Q Q^{*}\right)^{-1}, \\
& D^{\eta-1} Q_{k}^{*} D^{(k)} \text { and } D^{(k)} Q_{k} D^{\eta-1},
\end{aligned}
$$

which have exponential decay uniform in $k . D^{\eta}$ above is the modified Dirac operator on the $\eta=L^{-k}$ lattice. The kernels of the operators are related by

$$
\begin{aligned}
G_{k}^{\varepsilon}\left(L^{k} \varepsilon y, L^{k} \varepsilon y^{\prime}\right) & =\left(L^{k} \varepsilon\right)^{-(d-1)} G_{k}\left(y, y^{\prime}\right), \\
D^{(k), L^{k}}\left(L^{k} \varepsilon x, L^{k} \varepsilon x^{\prime}\right) & =\left(L^{k} \varepsilon\right)^{-(d+1)} D^{(k)}\left(x, x^{\prime}\right), \\
\Gamma^{(k), L^{k} \varepsilon}\left(L^{k} \varepsilon x, L^{k} \varepsilon x^{\prime}\right) & =\left(L^{k} \varepsilon\right)^{-(d-1)} \Gamma^{(k)}\left(x, x^{\prime}\right), \\
\left(D^{(0), \varepsilon-1} Q_{k}^{*} D^{(k), L^{k}}\right)\left(L^{k} \varepsilon y, L^{k} \varepsilon x\right) & =\left(L^{k} \varepsilon\right)^{-d}\left(D^{\eta-1} Q_{k}^{*} D^{(k)}\right)(y, x), \\
\left(D^{(k), L^{k}} Q_{k} D^{(0), \varepsilon-1}\right)\left(L^{k} \varepsilon x, L^{k} \varepsilon y\right) & =\left(L^{k} \varepsilon\right)^{-d}\left(D^{(k)} Q_{k} D^{\eta-1}\right)(x, y)
\end{aligned}
$$

for $y, y^{\prime} \in L^{-k} Z^{d}$ and $x, x^{\prime} \in Z^{d}$. We have

Theorem III.1. $\exists \beta>0, c>0$ independent of $k$, but dependent on a such that for sufficiently small $a$,
a) $\left|G_{k}\left(y, y^{\prime}\right)\right| \leqq c L^{k(d-1)} e^{-\beta\left|y-y^{\prime}\right|}$,
b) $\left|D^{(k)}\left(x, x^{\prime}\right)\right| \leqq c e^{-\beta\left|x-x^{\prime}\right|}$,
c) $\left|\Gamma^{(k)}\left(x, x^{\prime}\right)\right| \leqq c e^{-\beta\left|x-x^{\prime}\right|}$
d) $\left|D^{\eta-1} Q_{k}^{*} D^{(k)}(y, x)\right| \leqq c e^{-\beta|y-x|}$, 
e) $\left|D^{(k)} Q_{k} D^{\eta-1}(x, y)\right| \leqq c e^{-\beta|x-y|}$

for $y, y^{\prime} \in L^{-k} Z^{d}, x, x^{\prime} \in Z^{d}$.

Remark. The result $b$ ) doesn't hold uniformly in $a \leqq \infty$ as shown in the next section, i.e. doesn't hold for the " $\delta$-function" RGT.

We will prove Theorem III.1 using explicit momentum space formulas and the representations given by

$$
\begin{aligned}
G_{k} & =D^{\eta-1}-D^{\eta-1} Q_{k}^{*} D^{(k)} Q_{k} D^{\eta-1}, D^{(k)} \text { given by } \\
D^{(k)} & =a_{k}\left(I+a_{k} Q_{k} D^{\eta-1} Q_{k}^{*}\right)^{-1}, \\
\Gamma^{(k)} & =D^{(k)-1}-D^{(k)-1} Q^{*} D^{(k+1), L} Q D^{(k)-1}, D^{(k+1), L} \text { given by } \\
D^{(k+1), L} & =a L^{-1}\left(I+a L^{-1} Q D^{(k)-1} Q^{*}\right)^{-1},
\end{aligned}
$$

and obtained as in the proof of Lemma II.4.

We use the Fourier transform convention of [1]. Thus for $g \in l_{2}\left(L^{j} \in Z^{d}\right)$, $k \in l_{2}\left(\in Z^{d}\right)$, letting $\partial_{\mu}^{\alpha}(p)=\left(e^{i \alpha p_{\mu}}-1\right) / \alpha$,

$$
\left(Q_{j}^{*} g\right)^{\sim}(p+l)=\prod_{\mu=1}^{d} \frac{\overline{\partial_{\mu}^{L^{\prime}}(p+l)}}{\partial_{\mu}^{\varepsilon}(p+l)} \tilde{g}(p), \quad\left(Q_{j} k\right)^{\sim}(p)=\sum_{l} \prod_{\mu=1}^{d} \frac{\partial_{\mu}^{L^{J_{\varepsilon}}}(p+l)}{\partial_{\mu}^{\varepsilon}(p+l)} \tilde{k}(p+l),
$$

where

$p \in\left(-\pi / L^{j} \varepsilon, \pi / L^{j} \varepsilon\right]^{d}, l=2 \pi m / L^{j} \varepsilon, m \in Z^{d}$, such that $p+l \in(-\pi / \varepsilon, \pi / \varepsilon]^{d}, L$ odd. Thus

$$
\begin{aligned}
D^{\eta}(p) & =i \sum_{\mu=1}^{d} \gamma_{\mu} \frac{\sin \eta p_{\mu}}{\eta}+\eta \sum_{\mu} \frac{\left(1-\cos \eta p_{\mu}\right)}{\eta^{2}} \equiv i \gamma \cdot K(p)+M(p), \\
D^{\eta}(p)^{-1} & =(-i \gamma \cdot K(p)+M(p)) /\left(K(p)^{2}+M(p)^{2}\right) .
\end{aligned}
$$

We have

\section{Lemma III.1.}

a) $D^{(k)}\left(x, x^{\prime}\right)=\int \frac{d p}{(2 \pi)^{d}} D^{(k)}(p) e^{i p\left(x-x^{\prime}\right)}$

$$
=\int \frac{d p}{(2 \pi)^{d}} a_{k}\left(1+a_{k} \sum_{l} D^{\eta-1}(p+l) \prod_{\mu=1}^{d}\left|\frac{\partial_{\mu}^{1}(p+l)}{\partial \eta p(p+l)}\right|^{2}\right)^{-1} \cdot e^{i p\left(x-x^{\prime}\right)}
$$

b) $\Gamma^{(k)}\left(x, x^{\prime}\right)=\sum_{l} \int \frac{d p}{(2 \pi)^{d}} \frac{1}{a L^{-1}} D^{(k)}(p+l)^{-1} D^{(k+1), L}(p) e^{i(p+l)\left(x-x^{\prime}\right)}$

$$
+\sum_{l \neq l^{\prime}} \int \frac{d p}{(2 \pi)^{d}} D^{(k)}\left(p+l^{\prime}\right)^{-1} D^{(k+1), L}(p) D^{(k)}(p+l)^{-1} .
$$

$$
\left[\prod_{\mu}\left|\frac{\partial_{\mu}^{L}(p+l)}{\partial_{\mu}^{1}(p+l)}\right|^{2}-\prod_{\mu} \frac{\overline{\partial_{\mu}^{L}\left(p+l^{\prime}\right)}}{\partial_{\mu}^{1}\left(p+l^{\prime}\right)} \frac{\partial_{\mu}^{L}(p+l)}{\partial_{\mu}(p+l)} e^{-i\left(l-l^{\prime}\right) x^{\prime}}\right] e^{i l^{\prime}\left(x-x^{\prime}\right)} e^{i p\left(x-x^{\prime}\right)},
$$

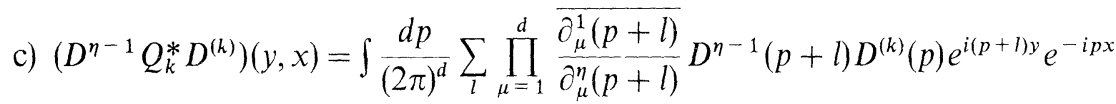


for $x, x^{\prime} \in Z^{d}, y \in L^{-k} Z^{d}, p \in(-\pi, \pi]^{d}, l, l^{\prime}=2 \pi m, m \in Z^{d}$ such that $p+l \in\left(-L^{k} \pi, L^{k} \pi\right]^{d}$ in a) and c); $p \in(-\pi / L, \pi / L]^{d}, l, l^{\prime}=(2 \pi m / L) m \in Z^{d}$ such that $p+l, p+l^{\prime} \in(-\pi \pi]^{d}$ in b).

Proof of Lemma III.1. The proofs of a) and c) are direct. b) follows as in [13] using the momentum space representation of

$$
D^{(k+1), L-1}=\left(a L^{-1}\right)^{-1} \cdot\left(I+a L^{-1} Q D^{(k)-1} Q^{*}\right) .
$$

The idea behind the proof of exponential decay is to show boundedness and analyticity of the integrands in Lemma III.1 in a small strip $T_{c}=\left\{\left|\operatorname{Im} p_{1}\right|<\alpha\right.$, $\left(\operatorname{Re} p_{1}, p_{2} \cdots p_{d} \varepsilon(-\pi, \pi]^{d}\right\}$, which results in exponential decay in the $\mu=1$ direction; by symmetry we have exponential decay in all directions. The proof of Theorem III.1 b)-e) can be carried out as in [13] using Theorem III.2 below which provides appropriate bounds and shows how the singularities in the integrands of Lemma III.1 get cancelled, the point being that a factor of $D^{\eta}(p)$ can be extracted. For the proof of Theorem III.1 a) use Theorem III.1 b)-e) and the decomposition formula of Theorem II.1 c) as in [33].

\section{Theorem III.2.}

a) $D^{(k)}(p)=a_{k} W(p)^{-1} D^{\eta}(p)=a_{k} D^{\eta}(p) W^{\prime}(p)^{-1}$,

$$
W(p)=D^{\eta}(p)+a_{k} \sum_{l} D^{\eta}(p) D^{\eta-1}(p+l) \prod_{\mu=1}^{d}\left|\frac{\partial_{\mu}^{1}(p+l)}{\partial_{\mu}^{\eta}(p+l)}\right|^{2},
$$

$W(p)$ analytic in $T_{c}, p \in(-\pi, \pi]^{d}, l=2 \pi m, m \in Z^{d}$,

$p+l \in\left(-L^{k} \pi, L^{k} \pi\right]^{d}$,

$$
|W(p)|<c, \quad\left|W(p)^{-1}\right|<c^{\prime} \quad p \in T_{c},
$$

and the same for $W^{\prime}(p)$.

b) $D^{(k+1), L}(p)=a_{k+1} L^{-1} V(p)^{-1} D^{\eta}(p)=a_{k+1} L^{-1} D^{\eta}(p) V^{\prime}(p)^{-1}$,

$$
V(p)=D^{\eta}(p)+a_{k+1} L^{-1} D^{\eta}(p) \sum_{l} D^{\eta-1}(p+l)\left|\frac{\partial_{\mu}^{L}(p+l)}{\partial_{\mu}^{\eta}(p+l)}\right|^{2},
$$

$V(p)$ analytic in $T_{c}, p \in\left(-\frac{\pi}{L}, \frac{\pi}{L}\right]^{d}, l=\frac{2 \pi m}{L}, \quad m \in Z^{d}$,

$p+l \in\left(-L^{k} \pi, L^{k} \pi\right]^{d}$,

$|V(p)|<c, \quad\left|V(p)^{-1}\right|<c^{\prime}, \quad p \in T_{c}$,

and the same for $V^{\prime}(p)$.

c) $D^{\eta}(p)$ analytic in $T_{c},\left|D^{\eta}(p)\right|<c$,

$D^{\eta}(p+l)^{-1}$ analytic in $p \in T_{c}$, for $l \neq 0$,

$\left|D^{\eta-1}(p+l)\right|<c(1+|p+l|)^{-1}$.

We defer the proof of Theorem III.2 to the end of the section.

The bounds in Theorem III. 2 can be proved using the following lemmas:

Lemma III.2. For $p \in T_{c}^{\prime}, K(p), M(p)$ and $\sin p / 2 / \eta^{-1} \sin \eta(p+l) / 2$ are analytic and have the bounds 

a) $\left|\frac{\sin \eta p / 2}{\eta^{-1} \sin \eta(p+l) / 2}\right| \leqq c(1+|l|)$,
b) $|K(p+l)|<c(1+|l|)$,
c) $|M(p+l)|<c(1+|l|)$.
$T_{c}^{\prime}=\left\{p: \operatorname{dist}\left(p_{1},(-\pi, \pi]\right)<\pi / 2,\left(p_{2}, \ldots p_{d}\right) \in(-\pi, \pi]^{d-1}\right\}$.

Proof of Lemma III.2. Follows from elementary estimates.

Lemma III.3. For $p \in T_{c}^{\prime \prime}\left(K^{2}+M^{2}\right)(p+l)$ is analytic and

$$
\left|\left(K^{2}+M^{2}\right)(p+l)\right| \geqq c\left(1+|p+l|^{2}\right), \quad l \neq 0 .
$$

$T_{c}^{\prime \prime}=\left\{p: \operatorname{dist}\left(p_{1},(-\pi, \pi]\right) \leqq r,\left(p_{2}, \ldots p_{d}\right) \in(-\pi, \pi]^{d-1}\right\}$.

Proof of Lemma III.3. We use the following result on the reciprocal of an analytic function: If $f(z)$ is analytic in $|z| \leqq R$, sup $|f(z)| \leqq M$ and $f(0)=m$, then $1 / f(z)$ is analytic in $|z| \leqq r \equiv(|m| / M) R / 4$ and $|1 / f(z)|<2 /|m|$ for $|z| \leqq r$. We apply this result in the complex variable $p_{1}$, for $l$ fixed, to the function, $K^{2}(p+l)+M^{2}(p+l)$ for circles of radius $R=\pi / 2$ in the complex $p_{1}$ plane centered at $p \in(-\pi, \pi]^{d}$. In $T_{c}^{\prime},\left(K^{2}+M^{2}\right)(p+l)$ is analytic and

$$
\left|\left(K^{2}+M^{2}\right)(p+l)\right|<4 c^{\prime}(1+|l|)^{2} .
$$

As we show below we have the lower bound

$$
\left|\left(K^{2}+M^{2}\right)(p+l)\right| \geqq c\left(1+|l|^{2}\right), \quad p \in(-\pi, \pi]^{d}, \quad c>0
$$

Thus

$$
\left|\left(K^{2}+M^{2}\right)(p+l)^{-1}\right| \leqq c_{2}\left(1+|l|^{2}\right)^{-1}
$$

in $T_{c}^{\prime \prime}$. For the lower bound use the $d$-dimensional equivalence of norms inequality

$$
\begin{aligned}
\frac{1}{\sqrt{ }}|\cdot|_{2} & \leqq|\cdot|_{1} \leqq \sqrt{ } d|\cdot|_{2} \text { to obtain, for }|\eta q| \leqq \pi, q \text { real, } \\
\left(K^{2}+M^{2}\right)(q) & \geqq \sum_{\mu} \frac{\sin ^{2} \eta q_{\mu}}{2}+\frac{1}{\eta^{2} d} \sum_{\mu}\left(1-\cos \eta q_{\mu}\right)^{2} \\
& =\left(1-\frac{1}{d}\right) \sum_{\mu} \frac{\sin ^{2} \eta q_{\mu}}{\eta^{2}}+\frac{1}{d \eta^{2}} \sum_{\mu} \sin ^{2} \eta_{\mu}+\frac{1}{d \eta^{2}} \sum_{\mu}\left(1-2 \cos \eta q_{\mu}+\cos ^{2} \eta q_{\mu}\right) \\
& \geqq \frac{1}{d} \sum_{\mu} \frac{2\left(1-\cos \eta q_{\mu}\right)}{\eta^{2}}=\frac{1}{d} \sum_{\mu} \frac{\sin ^{2} \frac{\eta q_{\mu}}{2}}{\left(\frac{\eta q_{\mu}}{2}\right)^{2} q_{\mu}^{2} \geqq \frac{4}{d \pi^{2}} \sum_{\mu} q_{\mu}^{2}}
\end{aligned}
$$

The lower bound above for $\left|\left(K^{2}+M^{2}\right)(p+l)\right|$ follows treating $l_{1} \neq 0$ and $l \neq 0$, separately. 


\section{Lemma III.4.}

a) $\left|\frac{\partial_{\mu}(p)}{\partial_{\mu}^{\eta}(p)}\right| \geqq \frac{2}{\pi}, \quad\left|\frac{\partial_{\mu}(p+l)}{\partial_{\mu}^{\eta}(p+l)}\right|<\frac{\pi}{2} \frac{\left|p_{\mu}\right|}{\left|p_{\mu}+l_{\mu}\right|}, \quad l_{\mu} \neq 0, \quad p \in(-\pi, \pi]$,

b) $|K(p)>c| p|, \quad \eta| p \mid>\frac{\pi}{2}$ p real, $\quad p \in(-\pi, \pi]^{d}, \quad c=\left(\frac{2}{\pi}\right)^{2}$,

c) $c^{\prime} q^{2}<K^{2}(q)+M(q)^{2}<c q^{2}, \eta|q| \leqq \pi$, q real,

$$
K^{2}(p+l)+M^{2}(p+l) \geqq \frac{1}{d \pi^{2}}|l|^{2}, \quad l=2 \pi m, \quad m \in Z^{d}, \quad p+l \varepsilon\left(-L^{k} \pi, K^{k} \pi\right]^{d} .
$$

Proof of Lemma III.4. The proofs of a) and b) are elementary; for c) the lower bound follows as in the proof of Lemma III.3. For the upper bound in c) write

$$
\begin{aligned}
M(q) & =\left(\sum_{\mu} \frac{2 \sin ^{2} \eta q_{\mu} / 2}{\eta}\right)^{2}, \text { so that }\left(K^{2}+M^{2}\right)(q) \leqq \pi q^{2}+\left(\sum_{\mu} \frac{1}{2} q_{\mu}^{2} \eta\right)^{2} \\
& \leqq q^{2}+q^{2}\left(\sum_{\mu} \frac{\left|q_{\mu}\right| \eta}{2}\right)^{2} \leqq\left(1+\left(\frac{d \pi}{2}\right)^{2}\right) q^{2} \text { as } \eta|q| \leqq \pi
\end{aligned}
$$

Proof of Theorem III.2a. For the upper bound on $W(p)$ use Lemma III.2, 3 and separate the $l=0$ term to get

$$
\begin{aligned}
|W(p)| \leqq & 2\left(|K(p)|^{2}+|M(p)|^{2}\right)^{1 / 2}+a_{k} c \\
& +a_{k} \sum_{l \neq 0} \frac{(|K(p)|+|M(p)|)(|K(p+l)|+|M(p+l)|)}{\left|\left(K^{2}+M^{2}\right)(p+l)\right|} \\
& \cdot \prod_{\mu=1}^{d}\left|\frac{\sin ^{2} p_{\mu} / 2}{\sin ^{2} \eta\left(p_{\mu}+l_{\mu}\right) / 2}\right|^{2} \frac{1}{(\eta / 2)^{-2}} \\
\leqq & +a_{k} c+\sum_{l \neq 0} \prod_{\mu=1}^{d} \frac{c}{\left(1+\left|l_{\mu}\right|\right)^{2}} \leqq c+\left(\sum_{l_{1}} \frac{c}{\left(1+\left|l_{1}\right|\right)^{2}}\right)^{d}<c .
\end{aligned}
$$

For the lower bound we use the analytic inverse operator theorem, the operator version of the reciprocal function theorem, stated in the proof of Lemma III.3. Note that $W(p)=R(p) I+A(p)$, where $R(p)$ is a real number and $A(p)$ is antihermitian. It is enough to bound $S p W(p)$, the spectrum of $W(p)$, away from zero for $p \in(-\pi, \pi]^{d}$. We do this by bounding $\operatorname{Re} S p W(p)$ away from zero for small $|p|$ using the $R(p)$ term only and separating out the $l=0$ term. For all $p$ and in particular for non-small $|p|$ we use the anti-hermitian terms to bound $\operatorname{Im} \operatorname{Sp} W(p)$ away from zero. Using Lemma III.4 a, c we have, letting $a_{k}=a^{\prime}$,

$$
\begin{aligned}
|\operatorname{Re} S p W(p)|= & \left.\left|M(p)+a^{\prime} \sum_{l} \frac{K(p) \cdot K(p+l)+M(p) M(p+l)}{\left(K^{2}+M^{2}\right)(p+l)} \prod_{\mu=1}^{d}\right| \frac{\partial_{\mu}^{1}(p+l)}{\partial_{\mu}^{n}(p+l)}\right|^{2} \mid \\
\geqq & M(p)+a^{\prime} c-a^{\prime} \sum_{l \neq 0} \frac{\left(K(p)^{2}+M(p)^{2}\right)^{1 / 2}\left(K(p+l)^{2}+M(p+l)^{2}\right)^{1 / 2}}{\left(K^{2}+M^{2}\right)(p+l)} \\
& \cdot \prod_{\mu=1}^{d} \frac{c}{\left(1+\left|l_{\mu}\right|\right)^{2}} \\
\geqq & a^{\prime} c-a^{\prime} c_{1}|p|>c a^{\prime} / 2,
\end{aligned}
$$


where we take $|p| \leqq c / 2 c_{1}$.

Now consider the anti-Hermitian terms (letting $\sigma_{\mu v}=\gamma_{\mu} \gamma_{v}-\gamma_{v} \gamma_{\mu}$ ),

$$
\begin{aligned}
A(p)= & i \gamma \cdot K(p)+i \gamma \cdot K(p) a^{\prime}\left(\sum_{l \neq 0} \frac{M(p+l)}{\left(K^{2}+M^{2}\right)(p+l)} \prod_{\mu=1}^{d}\left|\frac{\partial_{\mu}^{1}(p+l)}{\partial_{\mu}^{\eta}(p+l)}\right|^{2}\right) \\
& +i \gamma \cdot(-) M(p) a^{\prime} \sum_{l \neq 0} \frac{K(p+l)}{\left(K^{2}+M^{2}\right)(p+l)} \prod_{\mu=1}^{d}\left|\frac{\partial_{\mu}^{1}(p+l)}{\partial_{\mu}^{\eta}(p+l)}\right|^{2} \\
& +a^{\prime} \sum_{l \neq 0} \sum_{\mu, v} \frac{\sigma_{\mu \nu}}{2} \frac{\left(K_{\mu}(p) K_{v}(p+l)-K_{v}(p) K_{\mu}(p+l)\right)}{\left(K^{2}+M^{2}\right)(p+l)} \prod_{\mu=1}^{d}\left|\frac{\partial_{\mu}^{1}(p+l)}{\partial_{\mu}^{\eta}(p+l)}\right|^{2} \\
\equiv & i \gamma \cdot K(p)+i \gamma \cdot V(p)+\sigma \cdot F(p) .
\end{aligned}
$$

We treat $i \gamma \cdot V(p)+\sigma \cdot F(p)$ as a perturbation. We have

$$
|i \gamma \cdot V(p)+\sigma \cdot F(p)| \leqq a c_{1}|p|+a c_{2}|p|=a c_{3}|p|
$$

and the Neumann series of the resolvent $(A(p)-z)^{-1}$ converges for

$$
\left|(i \gamma \cdot K(p)-z)^{-1}\right||i \gamma \cdot V(p)+\sigma \cdot F(p)|<1 .
$$

Let $z=i \beta|p|$, then the above condition becomes

$$
\frac{1}{|K(p)|-\beta|p|} a c_{3}|p| \leqq \frac{a c_{3}|p|}{(c-\beta)|p|}<1,
$$

where we have used $|K(p)|>c|p|$. Taking $\beta \leqq c / 2$ and a such that $a \leqq c / 2 c_{3}$, we have convergence and thus $|\operatorname{Im} \operatorname{Sp} W(p)|>c / 2|p|$.

Combining the above results we have $|S p W(p)| \geqq c_{4}>0$ for all $p \in(-\pi, \pi]^{d}$, and $k$ so that $\left|W(p)^{-1}\right| \leqq 1 / c_{4}$. The region of analyticity of $W(p)$ is $T_{c}^{\prime \prime}$ but in applying the inverse operator theorem the region may shrink to $\left|p_{1}-\operatorname{Rep}_{1}\right| \leqq r / 4 c_{4} c \equiv \alpha$, $\left(\operatorname{Re} p_{1}, p_{2}, \ldots p_{d}\right) \in(-\pi, \pi]^{d}$, which contains $T_{c}$.

b) The proof is similar to a), c) follows from Lemma III.2.3.

\section{Non-Uniform Exponential Decay for the " $\delta$-Function" RGT}

In this section we show, contrary to RG intuition, that the sequence of rescaled actions associated with the " $\delta$-function" RGT does not have uniform exponential decay. The sequence of actions is obtained as the $a \rightarrow \infty$ limit of $D^{(k)}$ of Lemma III.2b and is given by

$$
\left(Q_{k} D^{\eta-1} Q_{k}^{*}\right)^{-1} \text {. }
$$

In one dimension we also show that the sequence of actions generated from an action of the form $e^{i \phi} p, \phi \in[0,2 \pi]$, display the same phenomena; occurring also for finite a for the actions $\pm p$.

The inverse of Eq. (4.1) has the explicit momentum space representation given 
by (see Lemma III.3a)

$$
\left(Q_{k} D^{\eta-1} Q_{k}^{*}\right)(p)=\sum_{l} D^{\eta-1}(p+l) \prod_{\mu=1}^{d}\left|\frac{\partial_{\mu}^{1}(p+l)}{\partial_{\mu}^{\eta}(p+l)}\right|^{2},
$$

where $p \in(-\pi, \pi]^{d}, l=2 \pi m, m \in Z^{d}, p+l \in\left(-L^{k} \pi, L^{k} \pi\right]^{d}$. The $k \rightarrow \infty$ limit of Eq. (4.2) is

$$
\sum_{l} \frac{-i \gamma \cdot(p+l)}{(p+l)^{2}} \prod_{\mu=1}^{d}\left|\frac{e^{i p_{\mu}}-1}{p_{\mu}+l_{\mu}}\right|^{2} \equiv-i \gamma \cdot f(p) .
$$

We show that $\left.f_{\mu}(p)\right|_{p_{t}= \pm \pi}=0$, so that for all $p$ with $p_{v}= \pm \pi$, for all $v$ we have $\gamma \cdot f(p)=0$, and thus the limit action $(-i \gamma \cdot f(p))^{-1}$ does not even exist at these points. To see this we sum over $l_{\mu}$ first in $f_{\mu}$ to get

$$
f_{\mu}(p)=\sum_{l_{v} \neq l_{\mu}} \prod_{v \neq \mu}\left|\frac{e^{i p_{\mu}-1}}{p_{v}+l_{v}}\right|^{2}\left|e^{i p_{\mu-1}}\right|^{2} \sum_{l_{\mu}} \frac{1}{\left(p_{\mu}+l_{\mu}\right)} \frac{1}{\left(p_{\mu}+l_{\mu}\right)^{2}+b^{2}},
$$

where $b^{2}=\sum_{\nu \neq \mu}\left(p_{v}+l_{v}\right)^{2}$. But for $p_{\mu}= \pm \pi$ the $l_{\mu}$ sum is

$$
\frac{1}{(2 \pi)^{3}} \sum_{n=-\infty}^{\infty} \frac{1}{\left(\frac{1}{2}+n\right)\left[\left(\frac{1}{2}+n\right)^{2}+\left(\frac{b}{2 \pi}\right)^{2}\right]}=0
$$

as the $n=0$ to $\infty$ sum is minus the $n=-1$ to $-\infty$ sum.

We now consider the exponential RGT in one dimension. Take

$$
D^{\eta}=e^{i \phi} \frac{\left(e^{i n p}-1\right)}{i \eta}
$$

as the original action and consider $D^{(\infty)}$, the $k \rightarrow \infty$ limit of the sequence of actions

$$
D^{(k)}=a_{k}\left(1+a_{k} Q_{k} D^{\eta-1} Q_{k}^{*}\right)^{-1} .
$$

Thus as $\lim _{k \rightarrow \infty} D^{\eta}(p) \equiv D^{c}(p)=e^{\imath \phi} p$, we have, with $a^{\prime}=\lim _{k \rightarrow \infty} a_{k}=\left(1-L^{-1}\right) a$,

$$
D^{(\infty)}(p)=a^{\prime}\left(1+a^{\prime} e^{-l \phi} \sum_{l} \frac{\left|e^{i p}-1\right|^{2}}{(p+l)^{3}}\right)^{-1}=\frac{a^{\prime}}{\left(e^{i \phi} p+a^{\prime} p f(p)\right)} D^{c}(p),
$$

where $f(p)=2(1-\cos p) \sum_{l}(p+l)^{-3}$.

The function $h(p)=p f(p)=(p / 2)^{-2} \sin ^{2}(p / 2) g(p), g(p)=\left[1+p^{3} \sum_{l \neq 0}(p+l)^{-3}\right]$

has the properties: $h(0)=1, h(\pi)=0, h(p)=h(-p)$, and $h(p)$ is monotone decreasing on $[0, \pi]$, since $(p / 2)^{-2} \sin ^{2}(p / 2)$ and $g(p)\left(g^{\prime}(p) \leqq 0\right.$ on $\left.[0, \pi]\right)$ are.

Thus for $\phi \neq 0, \pi\left(\phi=\pi / 2\right.$ corresponds to the Dirac action) $D^{(\infty)}(p)$ has analyticity and exponential falloff for all $a^{\prime} \in(0, \infty)$. However for $\phi=0, \pi$ boundedness and analyticity is lost for every $a^{\prime} \in(0, \infty]$. 


\section{Concluding Remarks}

Our results can be extended to include a mass term in the action. We are presently extending our results to the case of the Dirac field minimally coupled to a small external electromagnetic field, which can be considered as a step towards a block $\mathrm{RG}$ approach to QED. The $a \rightarrow \infty$ telescopic decomposition discussed in the introduction holds in a much more general context since it only involves the original operator and an appropriate averaging operator. Its analytical usefulness depends on the exponential decay of the difference of successive terms which has to be verified. In particular the decomposition applies to $\partial \Delta^{-1} \partial^{*}$, the gauge invariant two-point function of the free electromagnetic field.

Also several interesting spectral problems arise, i.e. what starting actions give rise to a sequence of rescaled actions which have uniform exponential decay and what is the role of positivity and self-adjointness?

\section{References}

1. Balaban, T.: (Higgs) ${ }_{2,3}$ quantum fields in a finite volume. I. A lower bound. Commun. Math. Phys. 85, 603-636 (1982)

2. Balaban, T.: (Higgs) ${ }_{2,3}$ quantum fields in a finite volume II. An upper bound. Commun. Math. Phys. 86, 555-594 (1982)

3. Balaban, T:: (Higgs) $)_{2,3}$ quantum fields in a finite volume III. Renormalization. Commun. Math. Phys. 89, 411-445 (1983)

4. Balaban, T.: Ultraviolet stability of three-dimensional lattice pure gauge field theories. Commun. Math. Phys. 102, 255-275 (1985)

5. Balaban, T.: Renormalization group approach to lattice gauge field theories I. Generation of effective actions in a small field approximation and a coupling constant renormalization in four dimensions. Commun. Math. Phys. 109, 249-301 (1987)

6. Balaban, T.: Renormalization group approach to lattice gauge theories. II. Analysis of effective densities and their expansions in the complete model. Physics Preprint, Harvard University Department 1987

7. Balaban, T., Imbrie, J., Jaffe, A.: Renormalization of the Higgs model: minimizers, propagators and the stability of mean field theory. Commun. Math. Phys. 97, 299-329 (1985)

8. Benfatto, G., Cassandro, M., Gallavotti, G., Nicoló, F., Oliviéri, E., Presutti, E., Scaciatelli, E.: Some probabilistic techniques in field theory. Commun. Math. Physics 59, 143-166 (1978)

9. Benfatto, G., Cassandro, M., Gallavotti, G., Nicolé, F., Oliviéri, E., Presutti, E., Scaciatelli, E.: Ultraviolet stability in Euclidean scalar field theories. Commun. Math. Phys.

10. Feldman J., Magnen J., Rivasseau, V., Sénéor, R.: The infrared behaviour of $\phi_{4}^{4}$

11. Feldman, J., Magnen, J., Rivasseau, V., Sénéor, R.: A renormalizable field theory: The massive Gross-Neveu model in two dimensions. Commun. Math. Phys. 103, 67-103 (1986)

12. Gallavotti, G., Nicoló, F.: Renormalizability in four-dimensional scalar fields. I. and II. Commun. Math. Phys. 100, 545-590 (1985); 101, 247-282 (1985)

13. Gawedzki, K., Kupiainen, A.: Block spin renormalization for dipole gas and $(\nabla \phi)^{4}$. Ann. Phys. 197, 198-243 (1983)

14. Gawedzki, K., Kupiainen, A.: Massless lattice $\phi_{4}^{4}$ theory: Rigorous control of a renormalizable asymptotically free model. Commun. Math. Phys. 99, 197-252 (1985)

15. Gawedzki, K., Kupiainen, A.: Gross-Neveu model through convergent perturbation expansions. Commun. Math. Phys. 102, 1-30 (1985)

16. Glimm, J., Jaffe, A.: Positivity of the $\phi_{3}^{4}$ Hamiltonian. Fortschr. Phys. 21, 327-376 (1973)

17. Balaban, T., O'Carroll, M., Schor, R.: Infrared asymptotic freedom for perturbations of the electromagnetic field on a lattice. Conference on Mathematical Quantum Field theory. Montreal 1987 
18. Dimock, J.: Infrared asymptotic freedom for the pseudoscalar Yukawa model at the critical point. Commun. Math. Phys. 109, 379-395 (1987)

19. Battle, G., Federbush, P.: Ondelettes and phase cell cluster expansions, A vindication. Commun. Math. Phys. 109, 417-419 (1987)

20. Federbush, P.: A phase cell approach to Yang-Mills, Theory. [0]-[V], Mathematics Department University of Michigan, Preprints 1987

21. Feldman, J., Hurd, T., Rosen, L., Wright, J.: QED: A Proof of Renormalizability, Mathematics Department Preprint, University of British Columbia, August 1987

22. Gallavotti, G.: Renormalization theory and ultraviolet stability for scalar fields via renormalization group methods. Rev. Mod. Phys. 57, 471-562, (1985)

23. Rivasseau, V.: Constructive renormalization. In VIIIth International Congress on Mathematical Phys., Mebkbout, M., Sénéor, R. (eds.). Singapore: World Scientific 1987

24. Scaling and Self-Similarity in Physics. Frohlich, J. (ed.) Progress in Physics Boston: Birkhäuser 1984

25. Critical Phenomena, Random Systems. Gauge Theories. Parts I and II. Les Houches 1984 Session XLIII. Elsevier Science Publishers. Amsterdam, the Netherlands 1987

26. Osterwalder, K., Seiler, E.: Gauge theories on a lattice. Am. Phys. 110, 440-471 (1978)

27. Seiler, E.: Gauge theories as a problem of constructive quantum field theory and statistical mechanics. Lecture Notes in Physics, vol. 159. Berlin, Heidelberg, New York: Springer 1982

28. Wilson, K. G.: Quantum Chromodynamics on a Lattice. In: Quantum field theory and statistical mechanics, pp. 143-172, 1976 Cargese Lectures, Levy. M. Mitter, P. (eds). New York: Plenum Press 1977

29. Wilson, K. G.: Monte Carlo calculations of the lattice gauge theory. In: Recent developments in gauge theories, pp. 363-402. 1979 Cargese Lectures t'Hooft, G., Itzykson, C., Jaffe, A., Lehmann, H., Mitter, P., Singer, I., Stora, R. (eds), New York: Plenum Press 1980

30. Berezin, F.: The method of second quantization. New York: Academic Press 1966

31. Battle, G.: A block spin construction of ondelettes. Part I. Lemarié Functions. Commun. Math. Phys. 110, 601-615 (1987)

32. Battle, G.: A block spin construction of ondelettes. Part II. The QFT Connection. Commun. Math. Phys. 114, 93-102 (1988)

33. Balaban, T.: Regularity and decay of lattice Green's functions. Commun. Math. Phys. 89, 571-597 (1983)

Communicated by K. Gawedzki

Received May 6, 1988; in revised form September 20, 1988 
